\title{
COMPOSIÇÃO DA ICTIOFAUNA DE TRÊS RIACHOS DO ALTO RIO TOCANTINS - GO
}

\author{
Jean Carlos Miranda \& Rosana Mazzoni
}

Biota Neotropica v3 (n1) - http://www.biotaneotropica.org.br/v3n1/pt/abstract?article+BN00603012003

Recebido em 10/10/2002

Revisado em 20/02/2003

Publicado em 10/03/2003

\begin{abstract}
Laboratório de Ecologia de Peixes, Departamento de Biologia Animal e Vegetal - Universidade do Estado do Rio de Janeiro, Pavilhão Haroldo Lisboa da Cunha, 20559-900 Rio de Janeiro, RJ, Brasil.(e-mail: jecarmi@ bol.com.br ; mazzoni@uerj.br)
\end{abstract}

Autor para correspondência: Jean Carlos Miranda: jecarmi@bol.com.br

\begin{abstract}
Fish species composition is presented for three streams from the upper Tocantins River. Bimonthly samples were taken by electrofishing. Fish communities in the Água Boa, Acaba Saco and Cavalo streams were composed of 35, 28 and 36 species, respectively. Characiformes and Siluriformes were the most abundant among the four registered orders. Constancy analyses revealed that all streams had a high number of occasional and rare species. The high diversity was registered at Acaba Saco stream, followed by Cavalo and Água Boa streams.
\end{abstract}

Keywords: Amazonian Basin, Tocantins River, Serra da Mesa, Streams, Fish Community

\section{RESUMO}

É apresentada a composição da ictiofauna de três riachos do alto rio Tocantins. Foram realizadas amostragens bimestrais, por pesca elétrica. A ictiofauna dos córregos Água Boa, Acaba Saco e Cavalo foram compostas por 35, 28 e 36 espécies, respectivamente. Characiformes e Siluriformes foram as ordens mais representativas dentre as quatro registradas. Os resultados da análise da constância de ocorrência revelaram um grande número de espécies ocasionais e raras. A maior diversidade foi registrada no córrego Acaba Saco, seguido dos córregos Cavalo e Água Boa.

Palavras-chave: Bacia Amazônica, rio Tocantins, Serra da Mesa, Riachos, Comunidades de Peixes 


\section{INTRODUÇÃO}

O principal objetivo da ecologia de comunidades é o reconhecimento dos mecanismos e processos responsáveis pelas diferenças e similaridades entre as diversas comunidades (Angermeier \& Karr, 1984). As forças que modelam a estrutura de uma comunidade, entendida aqui como o número de espécies e suas abundâncias relativas, são aquelas que determinam quais espécies ocorrem juntas, que espécies são comuns, que espécies são raras e as interações entre elas (Allan, 1995). O estudo de comunidades envolve uma síntese dos fatores ambientais e das interações bióticas e a estrutura de uma comunidade pode ser analisada sobre vários aspectos, utilizando-se parâmetros como diversidade, riqueza e equitabilidade (Wootton, 1995).

Dentre os padrões mais abordados em ecologia de comunidades estão aqueles relacionados com a diversidade de espécies (número, identidade e as características das mesmas), bem como aqueles relativos às variações temporais de suas abundâncias. Neste contexto o alto rio Tocantins e o conjunto de riachos que compõem o sistema desta área é de extrema importância para o entendimento das questões ictiofaunísticas, visto que $50 \%$ de sua ictiofauna encontrase em estado precário de identificação taxonômica (Figueiredo et al., no prelo)

Este trabalho é produto do projeto Estudos Básicos sobre a Ictiofauna do Aproveitamento Hidrelétrico Serra da Mesa, Goiás (FURNAS/Serra da Mesa/ UFRJ - Convênio UERJ) que forneceu informações sobre a diversidade ictiofaunística da região permitindo a descrição de novas espécies (p. ex. Britski, 1997, Fisch-Muller et al., 2001; Rapp Py-Daniel \& Oliveira, 2001).

O objetivo deste trabalho é apresentar a composição de espécies, distribuição espacial e diversidade específica das comunidades de peixes dos córregos Acaba Saco, Cavalo e Água Boa (sistema fluvial do alto rio Tocantins).

\section{MATERIAIS E MÉTODOS}

\section{Área de Estudo}

O alto rio Tocantins, na região de Serra da Mesa, é uma área atualmente sob influência de um represamento para a produção de energia elétrica e, os riachos estudados no presente trabalho, córregos Acaba Saco, Cavalo e Água Boa encontram-se situados nas áreas adjacentes ao reservatório recém formado (Figura 1). A descrição dos córregos é apresentada a seguir:

\section{Córrego Água Boa (13³4'35'S; 48¹3'51”W)}

Afluente da margem esquerda do rio Tocantins à montante da barragem de Serra da Mesa. O trecho estudado é caracterizado por uma extensa zona de corredeiras com fundo pedregoso e, em alguns pontos, com água bastante turbulenta. A jusante desse trecho tem-se uma pequena área de poça, também com fundo pedregoso; a montante da zona de corredeira uma longa e larga zona de rápidos com substrato de cascalho. A cobertura vegetal é quase inexistente, exceto em uma área marginal da zona de corredeira.

\section{Córrego Acaba Saco (14031'16"S; 4856'6"W)}

Afluente da margem direita do rio Tocantins à montante da barragem de Serra da Mesa. O trecho estudado apresenta extensas zonas de rápidos com fundo pedregoso intercaladas por pequenos remansos de fundo arenoso. A vegetação marginal se limita a pequenas porções de remansos. O trecho amostrado não possui nenhuma cobertura vegetal caracterizando-se, portanto como uma área aberta.

\section{Córrego Cavalo (14'26'12"S; 48 34'53"W)}

Afluente da margem esquerda do rio Traíras à montante da barragem de Serra da Mesa. O trecho estudado se caracteriza por apresentar zonas intercaladas de rápidos com fundo rochoso, corredeiras e rápidos com fundo pedregoso e remansos de fundo areno-lodoso. A vegetação marginal é escassa e a cobertura vegetal é quase total (aproximadamente 90\%) no trecho amostrado.

\section{Programa de Amostragem}

Os peixes utilizados neste trabalho foram coletados bimestralmente, entre dezembro de 1995 e dezembro de 1999 , utilizando-se pesca elétrica. Em cada riacho era estabelecido um trecho amostral com aproximadamente 80 metros de extensão, e esse mesmo trecho foi mantido por todo o período de amostragem. O detalhamento da técnica e avaliação da eficiência amostral da pesca elétrica pode ser obtido em Mazzoni et al. (2000).

Todos os peixes capturados eram mantidos vivos em gaiolas flutuantes e medidos. Após esse procedimento os exemplares eram devolvidos na porção média do trecho amostral. Exemplares com identificação duvidosa eram fixados em formalina a $10 \%$ e mantidos como testemunhos. A identificação das espécies foi realizada mediante bibliografia especializada (p. ex., Burgess, 1989; Garavello, 1979; Géry, 1977; Menezes, 1976; Santos et al., 1984) e quando necessário o auxílio de especialistas de diferentes grupos taxonômicos. 


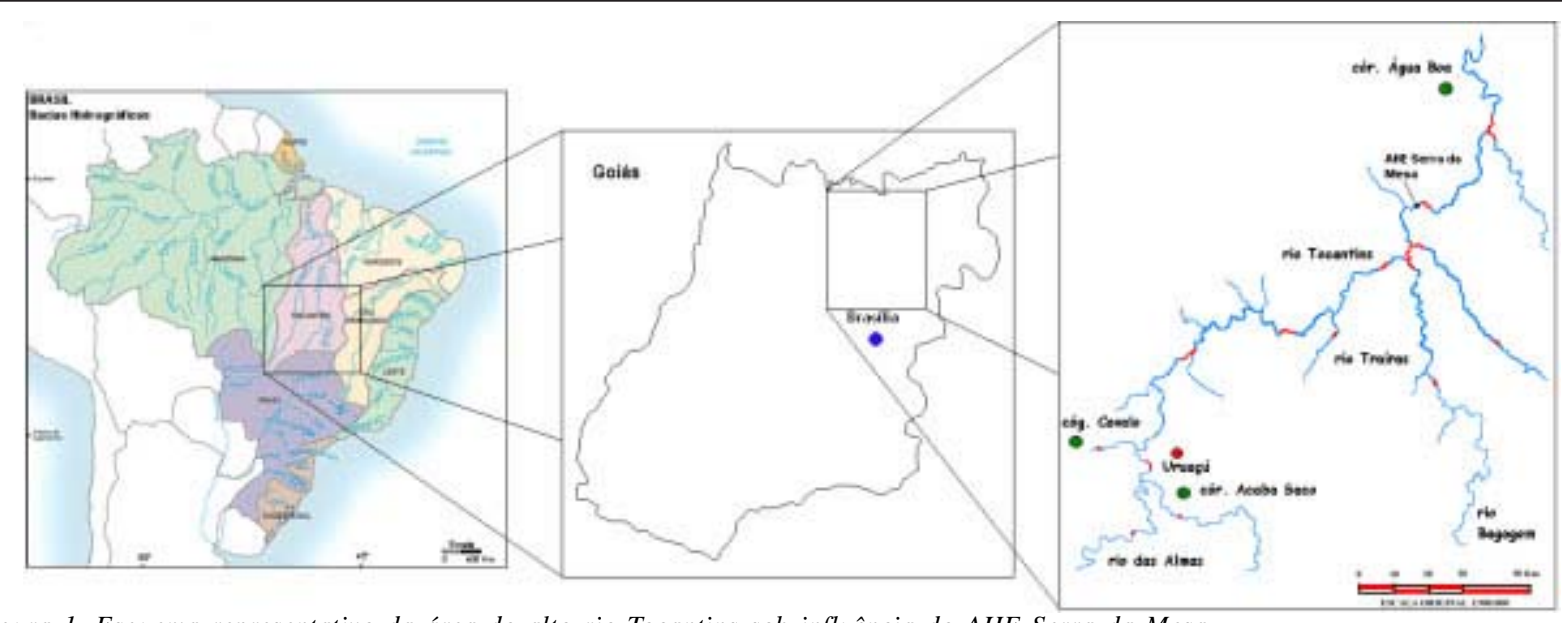

Figura 1. Esquema representativo da área do alto rio Tocantins sob influência do AHE Serra da Mesa.

\section{Tratamento dos dados}

A constância de ocorrência (C) das diferentes espécies foi determinada com base no percentual de períodos em que cada espécie ocorreu, sendo calculada de acordo com o modelo a seguir: $\mathrm{C}=\left(\mathrm{p}_{\mathrm{i}} \times 100\right) / \mathrm{P}$, onde: $\mathrm{p}_{\mathrm{i}}=$ número de coletas contendo a espécie i e $\mathrm{P}=$ total de coletas realizadas. Os valores calculados de $\mathrm{C}$ permitiram agrupar as espécies em três categorias: $(i)$ constantes $(\mathrm{C}>50 \%)$, (ii) acessórias (C>25<50\%) e (iii) acidentais ( $\mathrm{C}<25 \%)$. A fim de estabelecer o grau de dominância em cada comunidade, foi utilizado o Índice de Dominância (cf. McNaughton, 1968), calculado através da fórmula: $\mathrm{y} 1+\mathrm{y} 2 / \mathrm{Y}$, onde y $1=$ abundância da $1^{\mathrm{a}}$ espécie mais abundante, y2 = abundância da $2^{\mathrm{a}}$ espécie mais abundante e Y abundância total de todas as espécies.

A diversidade de espécies de cada riacho foi estabelecida para todo o período de estudo, bem como para cada mês de coleta separadamente; para tal utilizou-se o Índice de diversidade de Shannon-Wiener (McCune \& Mefford, 1999) e Equitabilidade de Pielou (Pielou - J = H' / H'max onde, H'max = In S onde, $\mathrm{S}=$ número de espécies) e seus resultados foram tratados comparativamente.

\section{RESULTADOS}

O córrego Acaba Saco tem sua ictiofauna composta por 28 espécies, o córrego Cavalo por 36 e o córrego Água Boa por 35 (Tabela 1). A representação gráfica da composição percentual de ordens representadas em cada riacho é apresentada na Figura 2.

No córrego Acaba Saco 12 espécies foram constantes, 8 acessórias e 8 acidentais; no córrego Cavalo 11 espécies foram constantes, 9 acessórias e 16 acidentais; no córrego Água Boa 13 espécies foram constantes, 7 acessórias e 15 acidentais (Tabela 2).
No que se refere à composição de espécies, tem-se que os três riachos estudados apresentam composição relativamente diferenciada. Considerando-se a totalidade das espécies amostradas (=47 spp.), 19 são comuns aos 3 riachos; no entanto, se forem consideradas apenas as espécies constantes e acessórias, este número é reduzido para $10(=21 \%)$, sendo elas: Astyanax gr. bimaculatus, Bryconamericus sp., Creagrutus sp., Harttia punctata., Hoplias gr. malabaricus, Hypostomus cf. plecostomus, Hypostomus sp.2, Knodus sp.5, Loricaria sp. e Phenacorhamdia sp. Os maiores números de espécies em comum, foram registrados entre os córregos Água Boa e Cavalo com 25 espécies em comum. O córrego Água Boa se destaca pelo maior número de espécies exclusivas (=6 spp.), são elas: Ancistrus aguaboensis, Apteronotus aff. albifrons, Aspidoras sp., Cochliodon sp., Eigenmannia cf. virescens e Sternopygus macrurus.

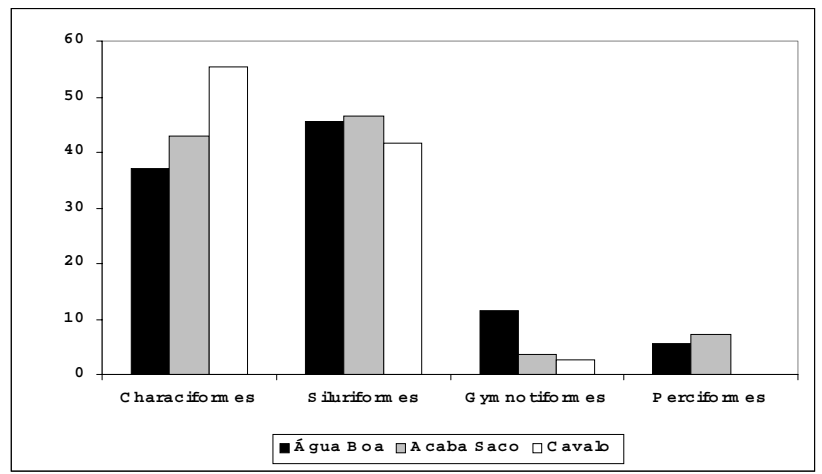

Figura 2. Composição percentual de ordens representadas em cada riacho analisado. 


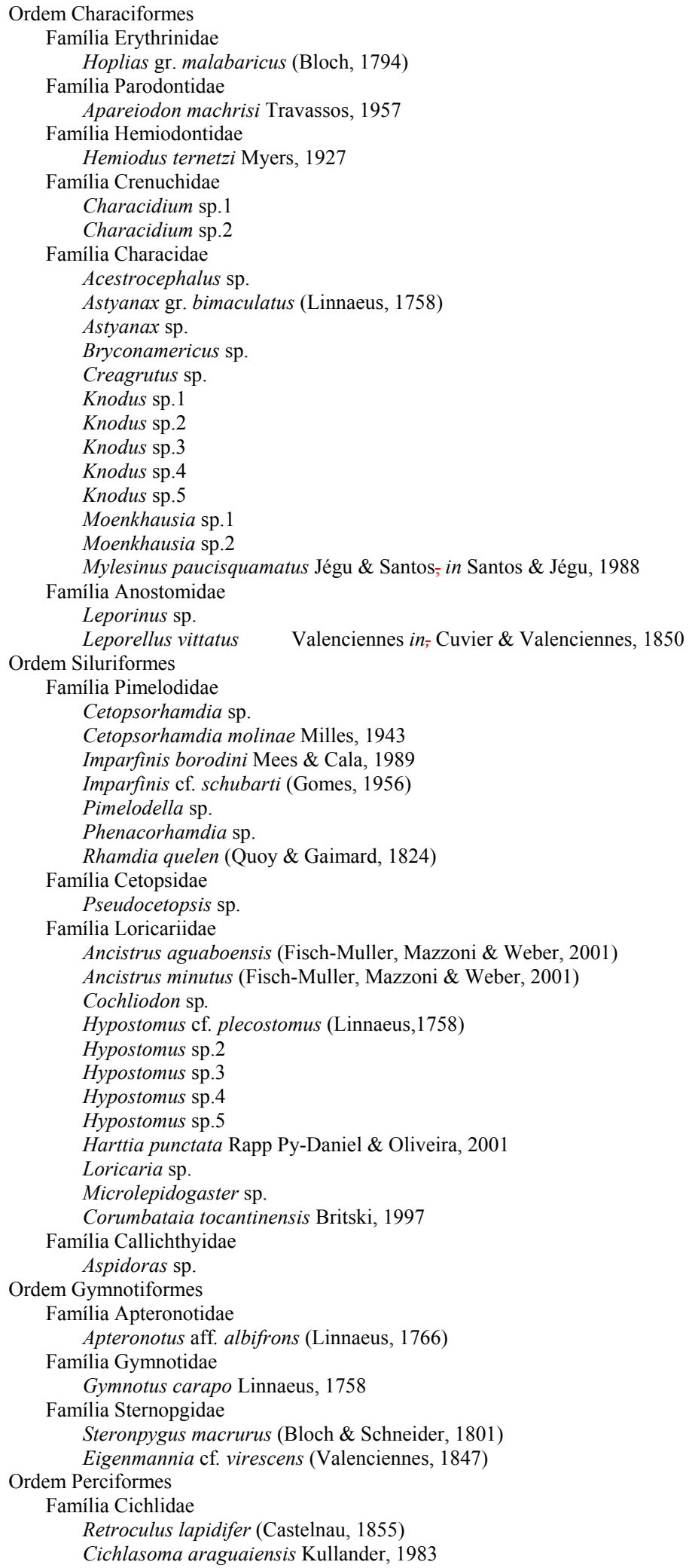

Tabela 1. Espécies de peixes coletadas nos córregos nos Córregos Água Boa, Acaba Saco e Cavalo, na região do AHE Serra da Mesa, alto rio Tocantins, GO. 


\begin{tabular}{|c|c|c|c|c|c|c|c|c|c|}
\hline \multirow[t]{2}{*}{ Espécies / Riachos } & \multicolumn{3}{|c|}{ Água Boa } & \multicolumn{3}{|c|}{ Acaba Saco } & \multicolumn{3}{|c|}{ Cavalo } \\
\hline & Co & Ace & Aci & Co & Ace & Aci & Co & Ace & Aci \\
\hline Acestrocephalus sp. & & & $\mathrm{x}$ & & & & & $\mathrm{x}$ & \\
\hline Ancistrus aguaboensis & $\mathrm{x}$ & & & & & & & & \\
\hline Ancistrus minutus & & & & $\mathrm{x}$ & & & $\mathrm{x}$ & & \\
\hline Apareiodon machrisi & & & $\mathrm{x}$ & & & $\mathrm{x}$ & $\mathrm{x}$ & & \\
\hline Apteronotus aff. albifrons & $\mathrm{x}$ & & & & & & & & \\
\hline Aspidoras sp. & $\mathrm{x}$ & & & & & & & & \\
\hline Astyanax gr. bimaculatus & $\mathrm{x}$ & & & $\mathrm{x}$ & & & $\mathrm{x}$ & & \\
\hline Astyanax sp. & & & & & & $\mathrm{x}$ & & & $\mathrm{x}$ \\
\hline Cichlasoma araguaiensis & & $\mathrm{X}$ & & & $\mathrm{x}$ & & & & \\
\hline Bryconamericus sp. & $\mathrm{x}$ & & & & $\mathrm{x}$ & & $\mathrm{x}$ & & \\
\hline Cetopsorhamdia molinae & & & $\mathrm{x}$ & & & & & & $\mathrm{x}$ \\
\hline Cetopsorhamdia sp. & & & $\mathrm{x}$ & $\mathrm{x}$ & & & & & $\mathrm{x}$ \\
\hline Characidium sp.1 & & & $\mathrm{x}$ & $\mathrm{x}$ & & & $\mathrm{x}$ & & \\
\hline Characidium sp.2 & & & & & & $\mathrm{x}$ & & $\mathrm{x}$ & \\
\hline Cochliodon sp. & & & $\mathrm{x}$ & & & & & & \\
\hline Creagrutus sp. & $\mathrm{x}$ & & & & $\mathrm{x}$ & & $\mathrm{x}$ & & \\
\hline Eigenmannia cf. virescens & & & $\mathrm{x}$ & & & & & & \\
\hline Gymnotus carapo & & & $\mathrm{x}$ & & & $\mathrm{x}$ & & & $\mathrm{x}$ \\
\hline Harttia punctata. & $\mathrm{x}$ & & & $\mathrm{x}$ & & & $\mathrm{x}$ & & \\
\hline Hemiodus ternetzi & & & & & & & & & $\mathrm{x}$ \\
\hline Hoplias gr. malabaricus & & $\mathrm{x}$ & & $\mathrm{x}$ & & & & $\mathrm{x}$ & \\
\hline Microlepidogaster sp. & & & & & $\mathrm{x}$ & & & & \\
\hline Corumbataia tocantinensis & & & & $\mathrm{x}$ & & & & & \\
\hline Hypostomus cf. plecostomus & $\mathrm{x}$ & & & & $\mathrm{x}$ & & $\mathrm{x}$ & & \\
\hline Hypostomus sp.2 & $\mathrm{x}$ & & & $\mathrm{x}$ & & & $\mathrm{x}$ & & \\
\hline Hypostomus sp.3 & & & $\mathrm{x}$ & & $\mathrm{x}$ & & & $\mathrm{x}$ & \\
\hline Hypostomus sp.4 & & & $\mathrm{x}$ & & & $\mathrm{x}$ & & & $\mathrm{x}$ \\
\hline Hypostomus sp.5 & & $\mathrm{x}$ & & $\mathrm{x}$ & & & & & $\mathrm{x}$ \\
\hline Imparfinis borodini & & & & & & & & $\mathrm{x}$ & \\
\hline Imparfinis cf. schubarti & $\mathrm{x}$ & & & & & & & $\mathrm{x}$ & \\
\hline Knodus sp.1 & & & $\mathrm{x}$ & & & $\mathrm{x}$ & & $\mathrm{x}$ & \\
\hline Knodus sp.2 & & & $\mathrm{x}$ & & & & & & $\mathrm{x}$ \\
\hline Knodus sp.3 & & & & & & $\mathrm{x}$ & & & $\mathrm{x}$ \\
\hline Knodus sp.4 & & & & & & & & & $\mathrm{x}$ \\
\hline Knodus sp.5 & & $\mathrm{x}$ & & $\mathrm{x}$ & & & & $\mathrm{x}$ & \\
\hline Leporellus vittatus & & & & & & & & & $\mathrm{x}$ \\
\hline Leporinus sp. & & & & & & & & & $\mathrm{x}$ \\
\hline Loricaria $\mathrm{sp}$ & $\mathrm{x}$ & & & $\mathrm{x}$ & & & $\mathrm{x}$ & & \\
\hline Moenkhausia sp.1 & & $\mathrm{x}$ & & & & & & $\mathrm{x}$ & \\
\hline Moenkhausia sp.2 & & & $\mathrm{x}$ & & & & & & $\mathrm{x}$ \\
\hline Mylesinus paucisquamatus & & $\mathrm{x}$ & & & $\mathrm{x}$ & & & & $\mathrm{x}$ \\
\hline Phenacorhamdia sp. & $\mathrm{x}$ & & & $\mathrm{x}$ & & & $\mathrm{x}$ & & \\
\hline Pimelodella sp. & & & & & & & & & $\mathrm{x}$ \\
\hline Pseudocetopsis sp. & & $\mathrm{x}$ & & & $\mathrm{x}$ & & & & \\
\hline Retroculus lapidifer & $\mathrm{x}$ & & & & & $\mathrm{x}$ & & & \\
\hline Rhamdia quelen & & & $\mathrm{x}$ & & & & & & $\mathrm{x}$ \\
\hline Sternopygus macrurus & & & $\mathrm{x}$ & & & & & & \\
\hline
\end{tabular}

Tabela 2. Classificação das espécies coletadas nos Córregos Água Boa, Acaba Saco e Cavalo de acordo com a constância de ocorrência . $C o=$ Constante, Ace $=$ Acessória, Aci $=$ Acidental . 


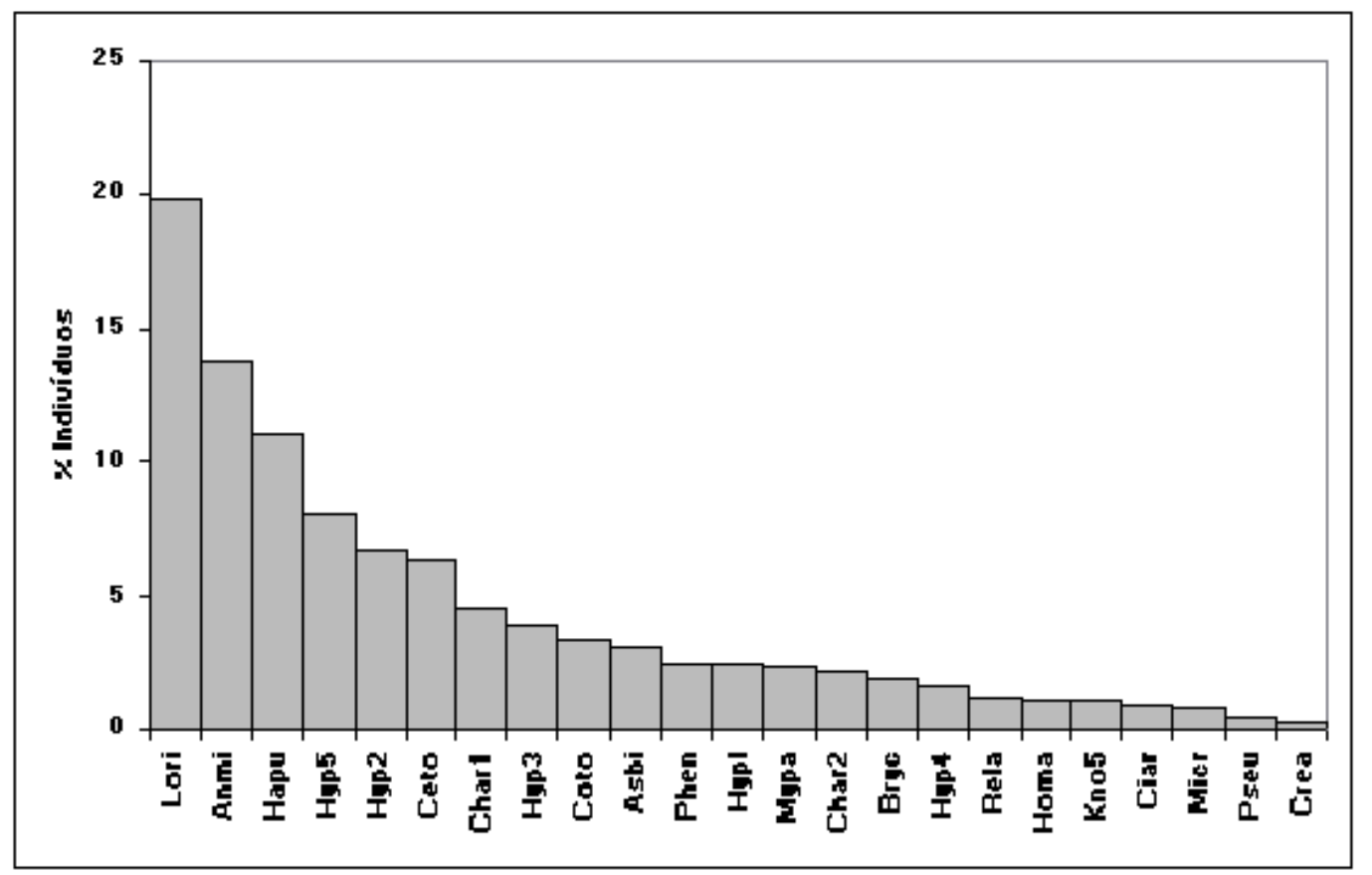

Figura 3. Distribuição dos valores percentuais das densidades médias das espécies de peixes do córrego Acaba Saco (Lori = Loricaria $s p$., Anmi = A. minutus, $\mathrm{Hapu}=\mathrm{H}$. punctata, $\mathrm{Hyp} 5=$ Hypostomus sp.5, Hyp $2=$ Hypostomus $s p 2$, Ceto $=$ Cetopsorhamdia $s p$. , Char $1=$ Characidium sp. 1, Hyp3 = Hypostomus sp.3, Coto = C. tocantinensis, Asbi = Astyanax gr. bimaculatus, Phen = Phenacorhamdia $s p$. . Hypl $=$ Hypostomus $c f$. plecostomus, Mypa $=$ M. paucisquamatus, Char $2=$ Characidium $s p .2$, Bryc $=$ Bryconamericus $s p$., Hyp $4=$ Hypostomus $s p .4$, Rela $=$ R. lapidifer, Homa $=$ Hoplias $g r$. malabaricus, Kno5 = Knodus sp.5, Ciar = C. araguaiensis, Micr $=$ Microlepidogaster $s p .$, Pseu $=$ Pseudocetopsis $s p$. . Crea $=$ Creagrutus $s p$.).

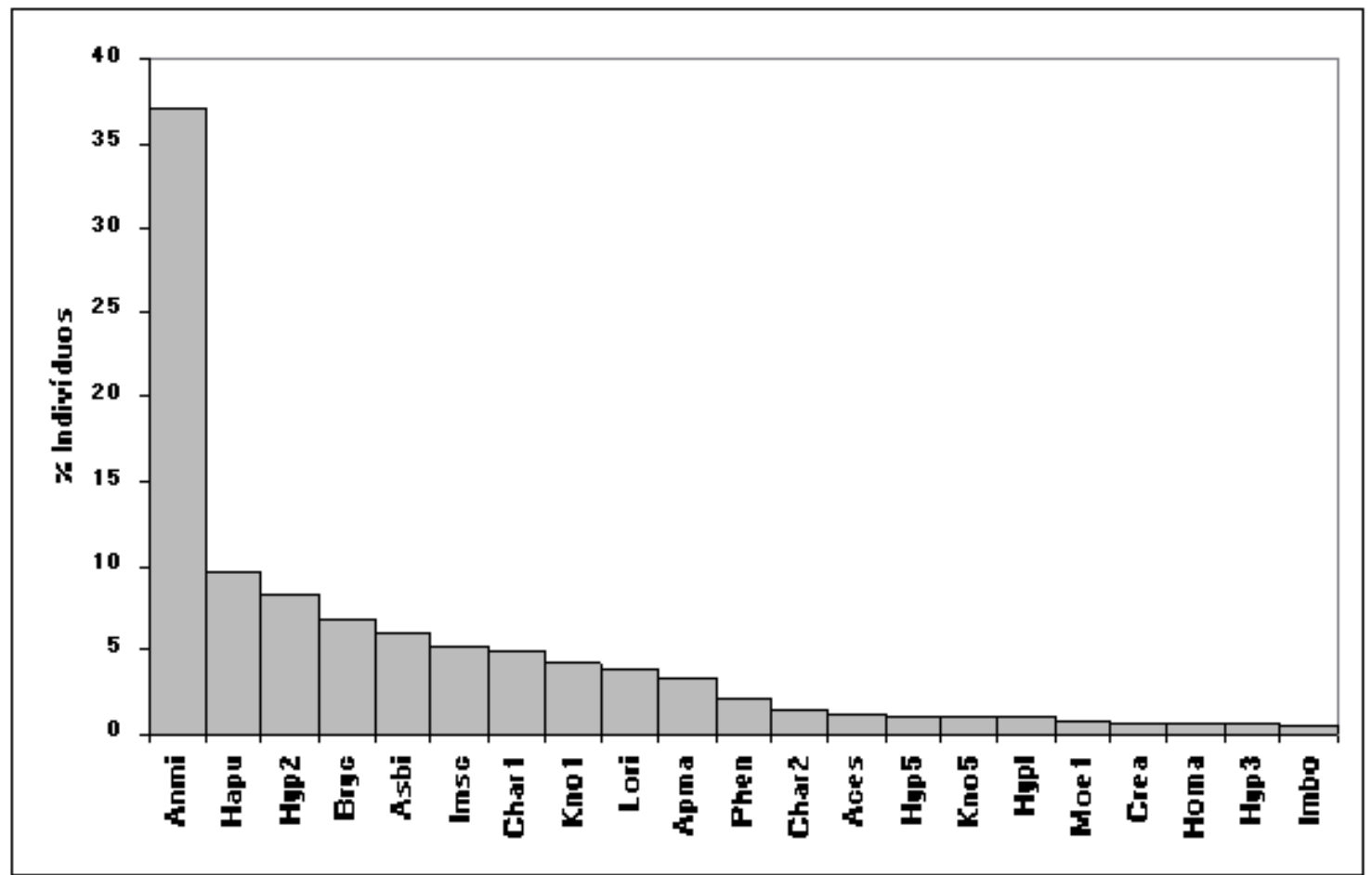

Figura 4. Distribuição dos valores percentuais das densidades médias das espécies de peixes do córrego Cavalo (Anmi = A. minutus, Hapu $=$ H. punctata, Hyp $2=$ Hypostomus $s p .2$, Bryc $=$ Bryconamericus $s p$. , Asbi = Astyanax $g r$ bimaculatus, Imsc = Imparfinis $g r$. schubarti, Charl $=$ Characidium sp.1, Knol $=$ Knodus sp.1, Lori $=$ Loricaria sp., Apma $=$ Apareiodon machrisi, Phen $=$ Phenacorhamdia sp., Char $2=$ Characidium sp.2, Aces $=$ Acestrocephalus sp., Hyp $5=$ Hypostomus sp.5, Kno5 $=$ Knodus $s p .5$, Hypl $=$ Hypostomus $c f$. plecostomus, Moe1 $=$ Moenkhausia sp.1, Crea $=$ Creagrutus sp., Homa $=$ Hoplias $g r$. malabaricus, Hyp3 $=$ Hypostomus sp.3, Imbo $=$ I. borodini).

http://www.biotaneotropica.org.br 


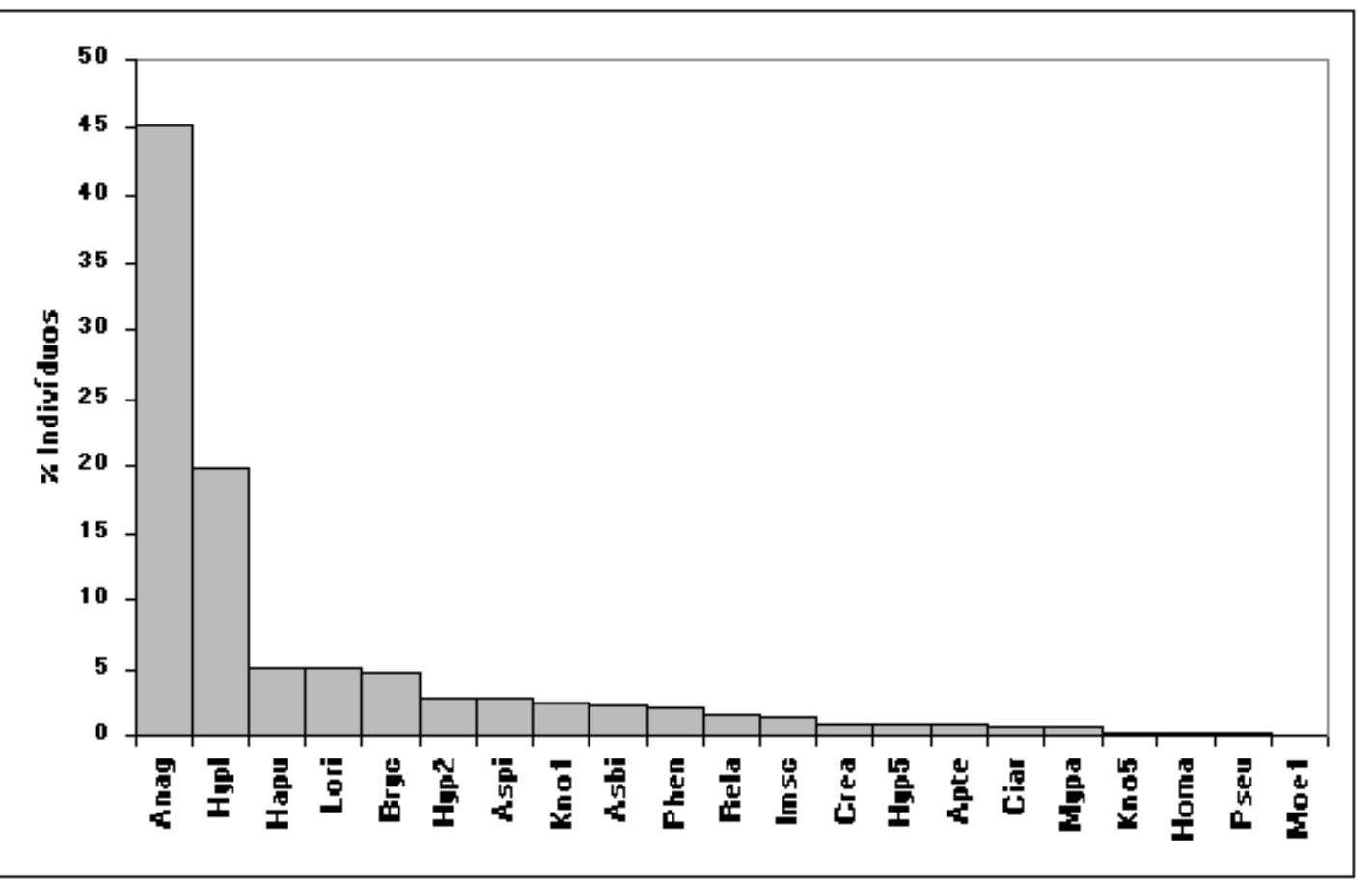

Figura 5. Distribuição dos valores percentuais das densidades médias das espécies de peixes do córrego Água Boa (Anag = A. aguaboensis, Hypl = Hypostomus $c f$. plecostomus, Hapu $=$ H. punctata, Lori = Loricaria $s p$., Bryc = Bryconamericus $s p$. , Hyp $2=$ Hypostomus sp.2, Aspi = Aspidoras sp., Knol = Knodus sp.1, Asbi = Astyanax gr bimaculatus, Phen = Phenacorhamdia sp., Rela = R. lapidifer, Imsc = Imparfinis $g r$. schubarti, Crea = Creagrutus sp., Hyp5 = Hypostomus sp.5, Apte = Apteronotus aff. albofrons, Ciar = C. araguaiensis, Mypa $=$ M. paucisquamatus, Kno5 = Knodus sp.5, Homa = Hoplias gr. malabaricus, Pseu = Pseudocetopsis $s p$. , Moel $=$ Moenkhausia sp.1).

\begin{tabular}{|l|c|c|c|}
\hline & Córrego Acaba Saco & Córrego Cavalo & Córrego Água Boa \\
\hline Riqueza (S) & 12,6 & 13 & 14,2 \\
\hline Diversidade (H') & 1,81 & 1,79 & 1,76 \\
\hline Equitabilidade & 0,73 & 0,71 & 0,66 \\
\hline
\end{tabular}

Tabela 3. Valores médios de riqueza, diversidade (índice de Shannon-Wienner) e equitabilidade das espécies de peixes dos córregos Acaba Saco, Cavalo e Água Boa no período entre dez/95 e dez/99. 
Os resultados do índice de constância indicam que, nos três riachos estudados, um elevado número de espécies tem ocorrência restrita (acidentais). Com base nesses resultados, determinamos as espécies consideradas residentes e, portanto, aquelas que efetivamente compõem as comunidades de peixes. Assim, apresentamos a representação gráfica da importância relativa das espécies que apresentaram ocorrência em no mínimo $25 \%$ das amostras e/ou que perfizeram $0,1 \%$ ou mais da densidade total estimada nos córregos Acaba Saco (Figura 3), Cavalo (Figura 4) e Água Boa (Figura 5).

Observa-se que o padrão de dominância é muito marcado para as três comunidades consideradas; no córrego Água Boa tem-se duas espécies com abundâncias superiores a $10 \%$ e as demais com importância reduzida; o Índice de Dominância é de 0,67, indicando que $67 \%$ dos exemplares da comunidade pertencem a duas únicas espécies, neste caso Ancistrus aguaboensis e Hypostomus cf. plecostomus. No córrego Acaba Saco, três espécies são dominantes, representando mais de $10 \%$ do total estimado; o Índice de Dominância é de 0,34, indicando que 34\% dos exemplares da comunidade pertencem a duas únicas espécies, neste caso Loricaria sp. e Ancistrus minutus. No córrego Cavalo, uma espécie é dominante, representando mais de $10 \%$ do total estimado; o Índice de Dominância é de 0,47 , indicando que $47 \%$ dos exemplares da comunidade pertencem a duas únicas espécies, neste caso Ancistrus minutus e Harttia punctata.

Na Tabela 3 são apresentados os valores médios da riqueza, diversidade e equitabilidade para as comunidades de cada riacho. Observa-se que a maior diversidade foi registrada no córrego Acaba Saco $\left(\mathrm{H}^{\prime}=1,81\right)$, seguido do Cavalo $\left(H^{\prime}=1,79\right)$ e Água Boa $\left(H^{\prime}=1,76\right)$; o padrão de riqueza média é distinto e, neste caso, o riacho com menor diversidade específica foi o que apresentou a maior riqueza. Tal fato está claramente explicado nos valores de equitabilidade; os menores valores de equitabilidade do córrego Água Boa justificam o padrão encontrado.

\section{DISCUSSÃO}

O predomínio de Siluriformes e Characiformes na ictiofauna dos três riachos estudados corrobora resultados encontrados por outros autores (p. ex. Lowe-McConnell, 1987; Mazzoni, 1998; Castro, 1999), de que Siluriformes e Characiformes são as ordens de maior representatividade na ictiofauna de sistemas fluviais sul-americanos.

Segundo Matthews (1998) uma interessante medida em estudo de comunidades de peixes, se refere ao número de famílias e ao número de espécies por família. O número de famílias representadas é relativamente grande em locais onde há grandes valores de riqueza (Moyle \& Li, 1979) e poucas famílias reúnem a maioria das espécies (Matthews, 1998). No presente estudo verificou-se que Characidae,
Loricariidae e Pimelodidae são as famílias com maior número de espécies, fato também demonstrado por outros autores (p. ex. Castro \& Casatti, 1997; Pavanelli \& Caramaschi, 1997; Bizerril, 1998; Uieda \& Barretto, 1999).

Muitas das espécies registradas neste trabalho (29 espécies) apresentam status taxonômico deficiente, o que revela o pouco conhecimento da ictiofauna da região e reforça a necessidade trabalhos taxonômicos da ictiofauna sul-americana, conforme já apontado por vários autores (Bölkhe et al., 1978; Menezes, 1992 e Reis, 1992). Mesmo depois de mais de duas décadas da publicação do trabalho de Bölkhe et al. (1978) ainda são relativamente poucos os trabalhos de cunho ecológico/naturalístico envolvendo ambientes fluviais de pequeno e médio porte (Castro, 1999). Esse fato explica os problemas freqüentes relativos aos limites taxonômicos de espécies de riachos da região Neotropical (Mazzoni, 1998; Bizerril, 1997, 1998; Pavanelli \& Caramaschi, 1997).

Gêneros tais como Ancistrus, Hypostomus e Characidium estão entre os táxons neotropicais mais especiosos, o que resulta em diversos problemas quanto aos reais limites taxonômicos de suas espécies (Géry, 1977; Burgess, 1989). Lowe-McConnell (1999) afirma que o estudo de ecologia de peixes em uma área tão vasta, remota e pouco explorada como a região Neotropical, é uma tarefa dificultada pela presença de muitas espécies, várias das quais muito semelhantes entre si, tornando muito difícil sua identificação, fato que explica a grande quantidade de morfotipos encontrados no presente estudo.

Suzuki et al. (1997) registraram 35 espécies em quatro tributários do reservatório de Segredo; Lobón-Cerviá et al. (1994) registraram 33 e 38 espécies em dois riachos (Campus e Barbará) do Pampa brasileiro; Garutti (1988) registrou 40 espécies em um córrego da bacia do Paraná. Os córregos Água Boa, Acaba Saco e Cavalo, bem como dos riachos citados, compõem bacias de grande porte, e seus elevados valores de riqueza corroboram com o proposto na literatura, de que o número de espécies que compõem a ictiofauna de um sistema é determinado pelas dimensões de sua bacia hidrográfica (Margalef, 1983).

Matthews (1998) afirma que riachos da região Neotropical apresentam maior riqueza de espécies que riachos de regiões temperadas, o que pode ser evidenciado quando comparados os valores de riqueza dos riachos por nós amostrados e alguns riachos de regiões temperadas. Por exemplo, considerando alguns estudos conduzidos em riachos da Inglaterra, Canadá, Estados Unidos da América do Norte, Polônia, Eslováquia e Dinamarca, foram registradas em torno de 9, 15, 11, 7,6 e 10 espécies de peixes, respectivamente (Le Cren, 1969; Mann, 1971; Mahon \& Balon, 1985; Neves \& Pardue, 1983; Penczak, 1981; Holcik, 1996; Mortensen, 1977). 
No que se refere à constância de ocorrência, registrou-se que as espécies com maior constância de ocorrência foram também as mais abundantes, resultado similar ao encontrado por Uieda (1984), Pavanelli \& Caramaschi (1997) para riachos da bacia do Paraná, Sabino \& Castro (1990) e Mazzoni \& Lobón-Cerviá (2000) para riachos costeiros do sudeste Brasileiro.

Muitos estudos sugerem que na maioria das comunidades animais há poucas espécies abundantes e muitas espécies representadas por poucos indivíduos (Matthews, 1998). Esta afirmação foi confirmada em nossos resultados onde registramos quatro espécies abundantes (Loricaria sp., Ancistrus minutus, Harttia sp. e Hypostomus sp5) no córrego Acaba Saco, quatro espécies (Ancistrus minutus, Harttia sp., Hypostomus sp.2 e Bryconamericus sp.) no córrego Cavalo e três espécies (Ancistrus aguaboensis, Hypostomus cf. plecostomus e Loricaria sp.) no córrego Água Boa.

O elevado número de espécies acidentais (8 no Acaba Saco, 16 no Cavalo e 15 no Água Boa) difere dos padrões registrados em outros riachos brasileiros. Somente Pavanelli \& Caramaschi (1997) registraram valores de espécies acidentais similares aos nossos. Garutti (1988) afirma que a constância de ocorrência é importante na caracterização de qualquer ponto de um curso d'água. É também uma medida qualitativa que pode evidenciar as espécies migrantes ou residentes de uma comunidade (Pavanelli \& Caramaschi, 1997), assim como o possível efeito das variações sazonais sobre as comunidades (Uieda, 1984).

Com base nos resultados de constância de ocorrência pode-se caracterizar duas ictiofaunas distintas, uma permanente (residente) e outra visitante (imigrante). LoweMcConnell (1975) afirma que uma comunidade residente pode ser aumentada por peixes imigrantes que se juntam aos residentes por algum tempo para se alimentar, reproduzir ou apenas passar pela comunidade. Garutti (1988) estudando a ictiofauna do córrego Barra Funda na bacia do Paraná, observou que $74 \%$ das espécies registradas crescem e reproduzem-se neste córrego, indicando um ambiente propício ao desenvolvimento ontogenético; os demais 26 $\%$ das espécies foram considerados visitantes. Com base nesse resultado o autor divide a comunidade de peixes em dois tipos de ictiofauna: uma residente e outra visitante e, sugere que esse mesmo resultado deve ser observado para outros sistemas fluviais. Estas informações são básicas para se analisar a importância dos pequenos afluentes na ecologia de rios de maior porte e o seu papel dentro do sistema global (Garutti, 1988).

Acreditamos que os resultados aqui apresentados, apesar do cunho informativo, refletem uma primeira interpretação da estrutura das comunidades de peixes dos riachos do alto rio Tocantins na área de influência do aproveitamento hidrelétrico Serra da Mesa. A continuidade deste estudo, incorporando informações sobre parâmetros populacionais talvez seja um dos caminhos para o entendimento sobre o funcionamento de comunidades de riachos na bacia amazônica.

\section{AGRADECIMENTOS}

Agradecemos a Érica Pellegrini-Caramaschi (UFRJ) e Ricardo Iglesias-Rios (UFRJ) pela coordenação do Projeto que deu origem aos dados desse trabalho. À todos os membros dos Laboratórios de Ecologia de Peixes da UERJ e da UFRJ pelo auxílio no trabalho do campo e processamento de parte do material. Esse trabalho foi realizado através do contrato Serra da Mesa Energia-Furnas/ BioRio/ UFRJ, convênio UERJ, e é parte da Dissertação do primeiro autor junto ao PPGB-Ecologia/UERJ.

\section{BIBLIOGRAFIA}

ALLAN, J.D. 1995. Stream Ecology - Structure and Function of Running Waters. Chapman \& Hall, New York.

ANGERMEIER, P.L. \& KARR, J.R. 1984. Fish communities along enviromental gradients in a system of tropical streams. Environ. Biol. Fishes 9: 117-135.

BIZERRIL, C.R.S.F. 1997. Mudanças temporais de comunidades ícticas em uma bacia hidrográfica do leste brasileiro. Com. Mus. Ciênc. Tecnol. 10: 53-75.

BIZERRIL, C.R.S.F. 1998. Comunidades de peixes do médio curso de sistemas fluviais da região carbonífera sulcatarinense. I. Bacia do rio Araranguá. Acta Biol. Leopol. 20: 225-242.

BÖHLKE, J.E., WEITZMAN, S. H. \& MENEZES, N. A. 1978. Estado atual da sistemática dos peixes de água doce da América do Sul. Acta Amazon. 8: 657-677.

BRITSKI, H.A. 1997. Descrição de duas espécies novas de Leporinus dos rios Araguaia e Tocantins, e comentários sobre as demais espécies do gênero assinaladas na bacia (Ostariophysi, Characiformes, Anostomidae). Com. Mus. Ciênc. Tecnol. 10: 27-43.

BURGESS, W.E. 1989. An Atlas of freshwater and marine catfishes. A preliminary survey of the Siluriformes. T.F.H. Publications, Neptune city.

CASTRO, R.M.C. 1999. Evolução da ictiofauna de riachos sul-americanos: padrões gerais e possíveis processos causais. In Ecologia de Peixes de Riachos (E.P. Caramaschi, E. P., R. Mazzoni. R. \& P.R. Peres-Neto, eds.). Série Oecologia Brasiliensis/PPGE-UFRJ, v.VI, p. 139-155.

CASTRO, R.M.C. \& CASATTI, L. 1997. The fish fauna from a small forest stream of the Paraná river Basin, southeastern Brazil. Ichthyol. Explor. Freshw.7: 337-352.

FIGUEIREDO, C.A.A., MORAES Jr., D.F., BARTOLETTE, 
R. \& PELLEGRINI-CARAMASCHI, E.P. no prelo. Composição taxonômica da ictiofauna da área da UHE Serra da Mesa. In Ictiofauna do Alto rio Tocantins Um Estudo de Caso e Subsídios para o Manejo no Reservatório de Serra da Mesa (R. Mazzoni, E. PellegriniCaramaschi \& R. Iglesias-Rios, eds.). Editora Copiarte, p. 000-000.

GARAVELLO, J.C. 1979. Revisão taxonômica do gênero Leporinus Spix 1829 (Ostariophysi, Anostomidae). Tese de doutorado, Universidade de São Paulo, São Paulo.

GARUTTI, V. 1988. Distribuição longitudinal da ictiofauna em um córrego da região noroeste do estado de São Paulo, bacia do rio Paraná. Rev. Brasil. Biol. 48: 747-759.

GERY, J. 1977. Characoids of the world. T.F.H. Publications.

HOLCIK, J. 1996. Ecological fish production in the inland delta of the middle Danube, a floodplain system. Env. Biol. Fish. 46: 151-156.

LE CREN, E.D. 1969. Estimates of fish population and production in small streams in England. In Symposium on Salmon and Trout in Streams (H.R. MacMillan Lectures in Fisheries). University of Britsh Columbia, p.269-280.

LOBÓN-CERVIÁ, J., UTRILLA, C. \& QUEIROL, H. 1994. An evaluation of the 3-removal method with electrofishing techniques to estimate fish number in streams of the brazilian Pampa. Arch. Hydrobiol. 130: 371-381.

LOWE-McCONNELL, R.H. 1975. Fish communities in tropical freshwaters: their distribution, ecology and evolution. Longman, London.

LOWE-McCONNELL, R.H. 1987. Ecological Studies in Tropical Fish Communities. Cambridge University Press, Cambridge.

LOWE-McCONNELL, R.H. 1999. Estudos ecológicos de comunidades de peixes tropicais. Edusp, São Paulo.

McNAUGHTON, S.J. 1968. Structure and function in California grassland. Ecology 49: 962-972.

McCUNE, B. \& MEFFORD, M. J. 1999. Multivariate analysis of ecological data, version 4, MjM Software Design. Gleneden Beach, Oregon.

MAHON, R. \& BALON, E.K. 1985. Fish production in warmwater streams in Poland and Ontario. Can. J. Fish. Aquat. Sci. 42: 1211-1215.

MANN, R.H.K. 1971. The population, growth and production of fish in four small streams in southern England. J. Anim. Ecol. 40: 155-190.

MARGALEF, R. 1983. Limnología. Ediciones Omega, Barcelona.

MATTHEWS, W.J. 1998. Patterns in Freshwater Fish Ecology. Chapman \& Hall, New York

MAZZONI, R. 1998. Estrutura da comunidades e produção de peixes de um sistema fluvial costeiro de Mata Atlântica, Rio de Janeiro. Universidade Federal de São Carlos. 100p.
MAZZONI, R. \& LOBÓN-CERVIÁ, J. 2000. Longitudinal structure, density and production rates of a neotropical stream fish assemblage: the river Ubatiba in the Serra do Mar, southeast Brazil. Ecography 23: 588-602

MAZZONI, R., FENERICH-VERANI, N. \& CARAMASCHI, E.P. 2000. Electrofishing as a sampling technique for coastal stream fish populations and communities in the Southeast of Brazil. Revista Brasileira de Biologia 60(2): 205-216

MENEZES, N. A. 1976. On the Cynopotaminae, a new subfamily of Characidae (Osteichthyes, Ostariophysi, Characoidei) Museu de Zoologia da Universidade de São Paulo 28, 91 p.

MENEZES, N. A. 1992. Sistemática de peixes. In: Agostinho, A. A. \& Benedito-Cecilio, E. (Eds.) Situação atual e perspectivas da ictiologia no Brasil - Documentos do IX Encontro Brasileiro de Ictiologia. Maringá- Editora da UEM. 128p.

MORTENSEN, E. 1977. Fish production in small Danish streams. Folia Limnologica Scandinavica 17: 21-26.

MOYLE, P.B. \& LI, H.W. 1979. Community ecolgy and predator-prey relationships in warmwater streams. 171-180. In: H. Clepper. Edt. Predator-prey systems in fisheries management. Sport Fishing Institute, Washington, D.C., Usa.

NEVES, R.J. \& PARDUE, G.B. 1983. Abundance and production of fishes in a small Appalachian stream. Trans.Am.Fish.Soc. 112: 21-26.

PAVANELLI, C.S. \& CARAMASCHI, E.P. 1997. Composition of the icthyofauna of two small tributaries of the Paraná river, Porto Rico, Paraná state, Brazil. Ichthyol. Explo. Fresh. 8: 32-31.

PENCZAK, T. 1981. Ecological fish production in two small lowland rivers in Poland. Oecologia 48: 107-111.

RAPP PY-DANIEL, L. H. \& OLIVEIRA, E. C. 2001. Seven new species of Harttia from the Amazonian-Guyana region (Siluriformes: Loricariidae). Ichthyol. Explor. Fresh., 12: 79-96.

REIS, R.E. 1992. Serão suficientes os sistematas de peixes neotropicais? In: Agostinho, A. A. \& Benedito-Cecilio, E. (Eds.) Situação atual e perspectivas da ictiologia no Brasil - Documentos do IX Encontro Brasileiro de Ictiologia. Maringá- Editora da UEM. 128p.

SABINO, J. \& CORRÊA ECASTRO, R.M. 1990. Alimentação, período de atividade e distribuição espacial dos peixes de um riacho da floresta Atlântica (sudeste do Brasil). Revista Brasileira de Biologia 50: 23-36.

SANTOS, G.M., JEGU, M. \& MERONA, B. 1984. Catálogo de peixes comerciais do baixo rio Tocantins. Eletronorte/ INPA 84p.

SUZUKI, H.I., PAVANELLI, C.S., FUGI, R., BINI, L.M. \& AGOSTINHO, A. A. 1997. Ictiofauna de quatro 
tributários do reservatório de Segredo. In: Agostinho, A. A. \& Gomes, L.C. (Eds). Reservatório de Segredo Bases ecológicas para o manejo. Maringá - EDUEM, p.259-273.

UIEDA, V. 1984. Ocorrência e distribuição dos peixes em riacho de água doce. Revista Brasileira de Biologia 44(2):203-213.

UIEDA, V. S. \& BARRETTO, M. G. 1999. Composição da ictiofauna de quatro trechos de diferentes ordens do rio Capivara, Bacia do Tietê, Botucatu, São Paulo. Rev. Bras. de Zoociências - Juiz de Fora v. 1 n. ${ }^{\circ} 1$ 55-67.

WOOTTON, R. J. 1995. Ecology of teleost fishes. Chapman \& Hall Press. 404 p.

Title: COMPOSIÇÃO DA ICTIOFAUNA DE TRÊS RIACHOS DO ALTO RIO TOCANTINS - GO

Authors: Jean Carlos Miranda \& Rosana Mazzoni

Biota Neotropica, Vol. 3 ( number 1): 2003

http://www.biotaneotropica.org.br/v3n 1/pt/ abstract?article+BN00603012003

Recebido em 10/10/2002 Revisado em 20/02/2003

Publicado em 10/03/2003

ISSN 1676-0603

http://www.biotaneotropica.org.br 